\title{
Pedigree-based assessment of recent population connectivity in a threatened rattlesnake
}

\author{
Scott Martin ${ }^{1}$, Greg Lipps ${ }^{1}$, and H. Lisle Gibbs ${ }^{2}$ \\ ${ }^{1}$ The Ohio State University \\ ${ }^{2}$ Ohio State University
}

January 21, 2021

\begin{abstract}
Managing endangered species in fragmented landscapes requires estimating dispersal rates between populations over contemporary timescales. Here we develop a new method for quantifying recent dispersal using genetic pedigree data for close and distant kin. Specifically, we describe an approach that infers missing shared ancestors between pairs of kin in habitat patches across a fragmented landscape. We then apply a stepping-stone model to assign unsampled individuals in the pedigree to probable locations based on minimizing the number of movements required to produce the observed locations in sampled kin pairs. Finally, we use all pairs of reconstructed parent-offspring sets to estimate dispersal rates between habitat patches under a Bayesian model. Our approach measures connectivity over the timescale represented by the small number of generations contained within the pedigree and so is appropriate for estimating the impacts of recent habitat changes due to human activity. We used our method to estimate recent movement between newly discovered populations of threatened Eastern Massasauga Rattlesnakes (Sistrurus catenatus) using data from 2996 RAD-based genetic loci. Our pedigree analyses found no evidence for contemporary connectivity between five genetic groups, but, as validation of our approach, showed high dispersal rates between sample sites within a single genetic cluster. We conclude that these five genetic clusters of Eastern Massasauga Rattlesnakes have small numbers of resident snakes and are demographically isolated conservation units. More broadly, our methodology can be widely applied to determine contemporary connectivity rates, independent of bias from shared genetic similarity due to ancestry that impacts other approaches.
\end{abstract}

\section{Hosted file}

SM_massassauga_connectivity_revised_clean2.pdf available at https://authorea.com/users/ 391005/articles/505216-pedigree-based-assessment-of-recent-population-connectivity-in-athreatened-rattlesnake 